Quim. Nova, Vol. 35, No. 3, 648-653, 2012

\title{
ESTUDIO DE USABILIDAD DE VISUALIZACIÓN MOLECULAR EDUCATIVA EN UN TELÉFONO INTELIGENTE
}

\author{
Miguel A. García-Ruiz* \\ Facultad de Telemática, Universidad de Colima, CP 28040, Colima, México \\ Laura L. Valdez-Velazquez y Zeferino Gómez-Sandoval \\ Facultad de Ciencias Químicas, Universidad de Colima, km 9, Colima-Coquimatlán, Colima, México
}

Recebido em 21/6/11; aceito em 5/9/11; publicado na web em 8/11/11

\begin{abstract}
A USABILITY STUDY OF EDUCATIONAL MOLECULAR VISUALIZATION ON SMART PHONES. Chemistry students have difficulty understanding molecular structures and their functions. To aide their comprehension, molecular visualization software has been developed to run on smart phones, but in order to positively influence learning it must have a high degree of usability (usability measures how software is used in terms of efficiency, efficacy and satisfaction). This paper describes a usability study of molecular visualization software running on a smart phone, where chemistry students analyzed molecular models. Results showed very good usability and $95 \%$ of students wanted to use it in further classes.
\end{abstract}

Keywords: molecular visualization; mobile computing; usability.

\section{INTRODUCCIÓN}

Los estudiantes de química generalmente tienen dificultad para comprender ciertos conceptos científicos de bioquímica y áreas afines, por ejemplo, las estructuras moleculares. ${ }^{1}$ Estudios empíricos llevados a cabo anteriormente ${ }^{2}$ han mostrado que muchos estudiantes no comprenden fácilmente las representaciones microscópicas de moléculas debido a su escala y sus características físico-químicas abstractas. La dificultad en el aprendizaje se debe principalmente a que suceden los procesos a una escala muy diferente y diminuta y con una estructura tridimensional demasiado intrincada, por lo que su comprensión resulta difícil. Esto sucede a menudo con la comprensión de los enlaces moleculares. ${ }^{3}$ A pesar de que los alumnos de química, en los primeros años de estudio de licenciatura, aprenden a cómo representar en diferentes formatos las geometrías moleculares (proyecciones de Fisher, taquigráfica, etc.) aún se requiere que el alumno muestre ciertas facultades espaciales para construir y entender la disposición tridimensional de las estructuras moleculares. ${ }^{4}$ Las herramientas didácticas tradicionales (por ejemplo, el pizarrón o los modelos moleculares de plástico) pueden servir para aprender ciertos conceptos generales sobre la estructura de las moléculas, pero tienen ciertas limitaciones para analizar y entender conceptos muy puntuales y precisos en la estructura y dinámica de las moléculas. Es por esto que para ayudar a su comprensión, los estudiantes deben apoyarse en información multisensorial, principalmente visual y táctil y dicha información debe mostrarse al estudiante de manera práctica y accesible. ${ }^{5}$

Una aplicación importante de la visualización molecular por computadora es en la enseñanza de las estructuras moleculares, de los elementos de simetría inherentes a las moléculas y algunas propiedades electrónicas que puedan visualizarse de acuerdo a algún código de colores, por ejemplo, densidad electrónica, el potencial electrostático, disposición de las fuerzas de van der Waals. ${ }^{6}$ Desde que se creó la WWW, los estudiantes de química pueden obtener de Internet infinidad de modelos moleculares en 3D, almacenados en formatos tales como VRML (Virtual Reality Modeling Language), el cual es un lenguaje de programación estándar para mundos

\footnotetext{
*e-mail: mgarcia@ucol.mx
}

virtuales, ${ }^{7}$ y en formato PDB (Protein Databank), ${ }^{8}$ entre otros. Los modelos moleculares bajados de Internet pueden ser visualizados con computadoras de escritorio utilizando programas visualizadores especiales, tales como el Rasmol y Jmol, entre muchos otros, los cuales pueden ser de fuente abierta (open source) y comerciales. ${ }^{9,10}$ La visualización científica (en particular la visualización molecular) por computadora ha sido utilizada ampliamente en las escuelas de todo el mundo en computadoras personales de escritorio y laptops. Canning et al..$^{11}$ entre otros han demostrado que la visualización molecular utilizada en el salón de clases ha apoyado grandemente la comprensión de aspectos abstractos de las estructuras moleculares, esto propicia que la enseñanza sea interactiva, entretenida y acorde a los gustos de los alumnos actuales donde su principal atención se encuentra en las computadoras, videojuegos y teléfonos móviles. El poder ver un compuesto en tres dimensiones en alguno de estos medios es una manera de acercarse al estudiante a su entorno; las imágenes de compuestos o las reacciones químicas no tienen ni idioma ni connotaciones culturales, por lo tanto, muchos recursos elaborados en otros países y en otros idiomas, se pueden utilizar sin tener que hacerles mayores cambios o traducirlos.

\section{VISUALIZACIÓN MOLECULAR EN DISPOSITIVOS MÓVILES}

Los dispositivos móviles tales como los teléfonos celulares son la herramienta digital más importante de comunicación de los jóvenes con una penetración del 66\% hasta marzo del 2011 en México (http://vivirmexico.com/2008/05/nuevas-estadisticas-telefonicasen-mexico).

Es innegable que el número de personas que usan teléfonos celulares con conexión a Internet (llamados también teléfonos inteligentes) en sus actividades diarias crece día con día, así como la demanda en interfaces gráficas interactivas más poderosas que desplieguen rápidamente información específica. Sin embargo, la aplicación de dispositivos móviles en la enseñanza de la química no es tan generalizada en México y en Latinoamérica, probablemente porque las estrategias o ideas que funcionan en una laptop no necesariamente son escalables o transferibles a dispositivos móviles. Sin embargo, con los navegadores de Internet de los teléfonos inteligentes actuales, 
Tabla 1. Lista de software de visualización molecular para dispositivos móviles

\begin{tabular}{lll}
\hline Nombre del software & Disponibilidad & Sistema operativo \\
\hline Mview & http://www.freewarepocketpc.net/ppc-download-mview-v1-35.html & Windows Mobile 2002/2003, corriendo en microprocesadores \\
& & ARM. \\
Pocketmol & http://birg.cs.wright.edu/pocketmol/pocketmol.html & Pocket PC con Windows CE o versión más reciente. \\
Molecules & http://itunes.apple.com/mx/app/molecules/id284943090?mt=8 & Compatible con iPhone, iPod touch y iPad de Apple. Requiere \\
& & iOS 3.1 o posterior.
\end{tabular}

los estudiantes pueden obtener mucha información química presente en la WWW. ${ }^{12}$

Recientemente, se ha desarrollado software para el apoyo de la educación química, incluyendo visualizadores de estructuras moleculares, para su aplicación en asistentes personales digitales (PDAs, por sus siglas en inglés) y otros dispositivos digitales móviles tales como los teléfonos inteligentes (también llamados smart phones). De acuerdo a Gilder et al..$^{13}$ la visualización molecular en dispositivos móviles puede ser útil para explorar de manera básica y práctica estructuras moleculares de proteínas, pudiendo utilizarse ésta en conferencias académicas, reuniones informales, sesiones de lluvia de ideas y otros tipos de reuniones donde no se tenga acceso inmediato a una computadora de escritorio. Complementando los hallazgos de Gilder et al., este tipo de visualización molecular móvil se puede realizar en cualquier lugar y en cualquier momento (anywhere-anytime) en línea y utilizando redes inalámbricas, permitiendo analizar y aprender aspectos generales de la forma y función de una molécula de manera rápida. Por ejemplo, un profesor de química puede explicar extra clase algún concepto de la estructura de una molécula a un alumno estando ambos fuera de clase en el pasillo o en la cafetería de la escuela. Es posible también utilizar estos visualizadores móviles en clase, para complementar alguna explicación dada por el profesor. Cabe mencionar que los programas de visualización molecular para dispositivos móviles generalmente son de bajo costo o gratuitos, ya que algunos son del tipo fuente abierta (open source software).

La Tabla 1 muestra una lista no exhaustiva de software para la visualización molecular que puede ser usado en teléfonos inteligentes.

Además de los visualizadores moleculares mostrados en la Tabla 1 , también es posible utilizar visualizadores de mundos virtuales para visualizar modelos de moléculas en dispositivos móviles. Un ejemplo de esto es el programa gratuito Pocket Cortona, disponible en http://www.parallelgraphics.com/products/cortonace/ . Con el Pocket Cortona los estudiantes pueden abrir un modelo en 3D interactivo de un átomo o molécula almacenada en formato VRML. La Figura 1 muestra el Pocket Cortona desplegando un modelo interactivo tridimensional de un átomo, utilizando un smart phone HP HW6940

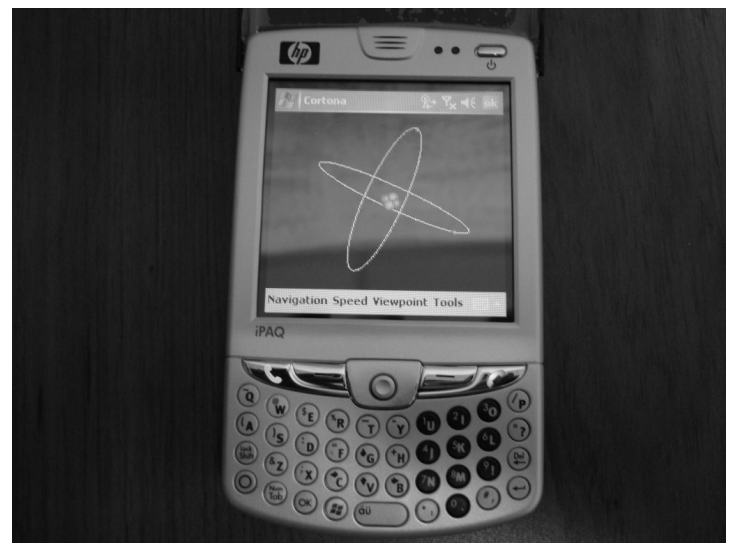

Figura 1. Visualizador Pocket Cortona mostrando el modelo en $3 D$ de un átomo corriendo Windows Pocket PC 2003. La Figura 2 muestra el programa Mview corriendo en dicho teléfono inteligente.

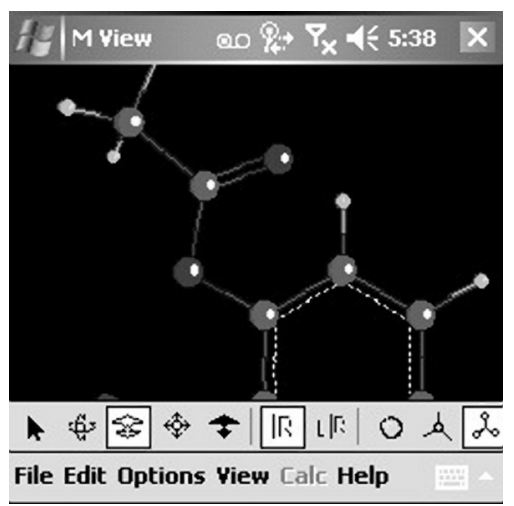

Figura 2. Visualizador Molecular Mview mostrando su menú principal y un modelo molecular

La visualización interactiva realizada en teléfonos inteligentes puede apoyar de manera favorable la comprensión de conceptos científicos en la enseñanza de la química ${ }^{14,15}$ tales como la comprensión de la estructura y funciones moleculares debido a su movilidad y disponibilidad en cualquier momento, entre otras razones, siempre y cuando las actividades educativas con el software de visualización se planeen de manera adecuada y se analice la usabilidad de dicho software para su mejora y para analizar qué tan útil y usable es dicho software para actividades pedagógicas. La usabilidad es un área de la Interacción Humano-Computadora que estudia la medida en que un producto puede ser utilizado con eficiencia, efectividad y satisfacción en un contexto de uso específico y donde el usuario cumple ciertas metas con el uso de dicho producto. ${ }^{16}$ Estudios recientes indican que si un programa de cómputo educativo tiene alto grado de usabilidad, éste apoyará de manera más efectiva el aprendizaje e impactará de manera positiva la motivación extrínseca de los estudiantes, entre otros beneficios educativos. ${ }^{17-19}$ Los resultados de las investigaciones anteriores también son consistentes con los resultados de usabilidad obtenidos por Virvou y Katsionis. ${ }^{20}$

\section{Estudio de usabilidad de visualización molecular en un teléfono inteligente}

Habiendo visto los recientes desarrollos de la visualización molecular en teléfonos inteligentes y la importancia e impacto de la usabilidad en el software educativo, nos dimos a la tarea de encontrar qué tan eficaz, eficiente y satisfactorio puede ser el uso de un visualizador molecular en un teléfono inteligente para el aprendizaje de estructuras moleculares para estudiantes de ciencias químicas de nivel superior (licenciatura).

Para analizar la usabilidad de un visualizador molecular en un teléfono inteligente utilizado en un entorno educativo, fue necesario conducir un estudio de usabilidad basado en el método de Think Aloud Protocol $^{21}$ en combinación con el llenado de un cuestionario 
de usabilidad llamado System Usability Scale (SUS). ${ }^{22}$ Estos métodos han sido ampliamente utilizados en la industria del software y también para analizar la usabilidad de software educativo. En el método de Think Aloud Protocol se le pide a un usuario que interactúe con la interfaz llevando a cabo una tarea (actividad). Mientras el usuario realiza la tarea éste debe hablar en voz alta y explicar lo que él o ella está pensando y percibiendo acerca de la interacción con el programa a ser evaluado. El especialista en usabilidad debe mantener hablando al usuario, haciéndole preguntas como "¿por qué seleccionaste esa opción?" o “¿qué opinas de los colores de la interfaz?”, pero sin interferir en la tarea del usuario. El cuestionario SUS se llena después de haber realizado las tareas. Este cuestionario nos permitirá analizar diez valores puntuales de la usabilidad de un producto de software y arrojará un valor de usabilidad general en una escala de 0 a 100, donde 0 significa que el producto no tiene usabilidad y 100 significa que el producto tiene usabilidad excelente.

\section{Preguntas de investigación}

Después de haber analizado la revisión de la literatura sobre visualización de modelos moleculares en ambientes educativos, surgen las siguientes preguntas de investigación:

1. ¿Los estudiantes podrán visualizar de manera satisfactoria la estructura molecular en la pantalla del teléfono inteligente?

2. ¿Los estudiantes podrán interactuar de manera satisfactoria con la estructura molecular mostrada en el teléfono inteligente?

3. ¿El visualizador molecular corriendo en el teléfono inteligente será lo suficientemente eficaz y eficiente para mostrar la molécula a estudiar?

4. ¿Los estudiantes estarán interesados en seguir utilizando el visualizador molecular en el teléfono inteligente en futuras clases?

\section{Participantes}

Veintidós estudiantes (13 mujeres y 9 hombres) de sexto semestre de la carrera de Químico Farmacéutico Biólogo de la Facultad de Ciencias Químicas de la Universidad de Colima, México, fueron invitados libremente a participar en nuestro estudio de usabilidad, cuyo promedio de edad es de 21 años, quienes previo al estudio de usabilidad cursaron las materias de química inorgánica y química orgánica. Los estudiantes declararon que todos ya habían utilizado visualizadores moleculares en computadoras de escritorio, principalmente el Hyperchem (http://www.hyper.com), pero ninguno había utilizado visualizadores moleculares en dispositivos móviles. Los participantes en el estudio de usabilidad fueron seleccionados y tratados con respecto al Código Ético de la Investigación en Usabilidad e Interacción Persona-Ordenador de la Asociación Interacción Persona-Ordenador (AIPO) de España, ${ }^{23}$ a falta de un código de ética local especializado en usabilidad.

\section{Materiales}

Para las pruebas de usabilidad se utilizó un teléfono inteligente marca Hewlett-Packard, modelo HW6940, corriendo Windows Pocket PC 2003 (Figura 1). Se utilizó además el programa Mview versión 1.4 para la visualización molecular (Figura 2), el cual fue instalado en el teléfono inteligente. Este programa se encuentra disponible en http://www.freewarepocketpc.net/ppc-download-mview-v1-35. html. Se escogió este software porque se puede bajar de Internet e instalar de manera gratuita. Mview permite abrir archivos en formato Protein Databank (PDB) y XYZ entre otros, permitiendo abrir miles de modelos moleculares descargables de la Internet. Al igual que otros visualizadores moleculares, el Mview permite rotar el modelo molecular usando las flechas del teclado, calcula el ángulo de torsión y muestra el modelo en formato "CPK", wireframe y ball and stick. Para este estudio de usabilidad, los participantes visualizaron las moléculas en formato ball and stick.

Los modelos moleculares utilizados por los estudiantes en el estudio de usabilidad fueron los siguientes (Figura 3): etano $\left(\mathrm{C}_{2} \mathrm{H}_{6}\right)$; alanina $\left(\mathrm{C}_{3} \mathrm{H}_{7} \mathrm{NO}_{2}\right)$ y buckminsterfullereno $\left(\mathrm{C}_{60}\right)$
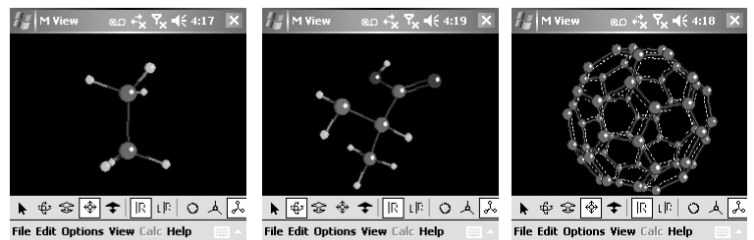

Figura 3. Screenshots del programa Mview desplegando los modelos moleculares del Etano, Alanina y Buckminsterfullereno, respectivamente

\section{¿Por qué utilizamos estas moléculas en el estudio de usabilidad?}

A pesar de que la selección de las moléculas en este estudio tiene una dosis de arbitrariedad, se consideraron algunos aspectos generales. En primer lugar se escogió al etano como una molécula ampliamente conocida y familiar por los alumnos del sexto semestre de la carrera de Químico Farmacéutico-Biólogo, ya que ha sido objeto de estudio desde la perspectiva de la teoría de enlace valencia y de los orbitales moleculares. Es una molécula de partida apropiada para determinar fácilmente las longitudes de enlace, ángulos de enlace y ángulos diedros.

Con respecto a la alanina, es uno de los veinte aminoácidos constitutivos de las proteínas y la de menor tamaño molecular que tiene un carbono $\alpha$ quiral y en principio es más fácil asignar la configuración $(R)-(S)$ por ser menos voluminosa que el resto de los aminoácidos.

El C ${ }_{60}$, llamado buckminsterfullereno o buckyball, es uno de los miembros de una familia de "estructuras cerradas" de carbono llamados fullerenos. ${ }^{24,25}$ Debido a la alta simetría y estructura fascinante del $_{60}$ los alumnos muestran una marcada curiosidad por este tipo de moléculas. Una de las características de la familia de los fullerenos es que todos tienen 12 caras pentagonales y pueden tener una gran variedad de caras hexagonales. De hecho, los fullerenos siguen la regla $\mathrm{C}_{20+2 \mathrm{H}}$ donde $\mathrm{H}$ es el número de caras hexagonales. ${ }^{26}$ Por esta razón se le preguntó al alumno en un cuestionario la cantidad de pentágonos que posee el buckyball.

\section{Procedimiento}

Se realizaron estudios de usabilidad de manera individual con cada participante de acuerdo al método Think Aloud Protocol (TAP). El estudio de usabilidad consistió en 4 fases. En la primera, se le explicó a cada estudiante el propósito y actividades a realizar en el estudio de usabilidad. En la segunda fase, el estudiante tuvo hasta 5 min para familiarizarse con el teléfono inteligente y el programa Mview interactuando con un modelo molecular sencillo (Etano). En la tercera fase, los estudiantes interactuaron con los modelos moleculares utilizando el programa Mview. En la cuarta fase el estudiante llenó el cuestionario de usabilidad mostrado en el Anexo A y una tabla de resultados.

Mediante una presentación en Power Point se les explicó a los estudiantes el objetivo del estudio de usabilidad con el teléfono inteligente, mostrando modelos de las moléculas de estudio y las tareas a realizar en este dispositivo. Además, en la presentación de Power Point se explicó el funcionamiento y menú del programa Mview. Acto seguido, se le dio a cada estudiante las instrucciones impresas de la presentación de Power Point para que el alumno tuviera a la mano el procedimiento.

A continuación cada uno de los estudiantes realizó las siguientes tareas en el teléfono inteligente:

1) Visualizar la molécula del etano en formato ball and stick y 
calcular: La longitud de enlace $\mathrm{C}-\mathrm{C}$ y $\mathrm{C}-\mathrm{H}$; $\mathrm{El}$ ángulo de enlace $\mathrm{C}-\mathrm{C}-\mathrm{H}$; El ángulo diedro $\mathrm{H}-\mathrm{C}-\mathrm{C}-\mathrm{H}$.

2) Visualizar la molécula de Alanina en formato ball and stick y determinar: El carbono quiral y

La configuración $(R)-(S)$ del estereocentro de acuerdo a la metodología de Cahn-Ingold-Prelog.

3) Visualizar el $\mathrm{C}_{60}$ en formato ball and stick y por medio de rotaciones determinar el número de pentágonos que posee.

Después de haber realizado los ejercicios anteriores cada estudiante contestó el cuestionario de usabilidad que se muestra en el Anexo A así como una tabla de resultados.

La Figura 4 muestra un participante utilizando el teléfono inteligente. En la foto se puede apreciar que el estudiante utiliza el lápiz apuntador del teléfono para seleccionar opciones del menú del programa Mview y para rotar y ampliar (hacer zoom) a los modelos moleculares, además de medir los ángulos de enlace.

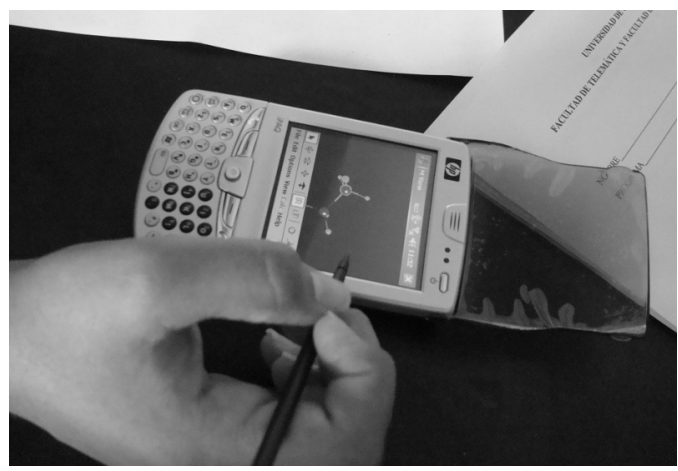

Figura 4. Un participante interactuando con un modelo molecular en el teléfono inteligente

\section{RESULTADOS}

El promedio de los resultados del cuestionario SUS fue de 86.3, dentro de una escala de valor de usabilidad 0 a 100 , propuesta por Brooke. ${ }^{22}$ Esto significa que la interacción con los modelos moleculares en el teléfono inteligente tuvo muy buena usabilidad (eficacia, eficiencia y satisfacción de uso). La Tabla 2 muestra los resultados de cada una de las 10 escalas de Likert del cuestionario SUS, donde $1=$ En completo desacuerdo y $5=$ Completamente de acuerdo. Se calculó el promedio y la moda estadística de los resultados de cada escala de los 22 participantes. La tabla también muestra la desviación estándar de cada escala, donde se ve una distribución de los datos aceptable.

Con respecto a las preguntas de investigación, se encontró lo siguiente:

1. ¿Los estudiantes podrán visualizar de manera satisfactoria la es-

tructura molecular en la pantalla del teléfono inteligente?

Ningún estudiante se quejó del tamaño de la pantalla del teléfono inteligente utilizado en el estudio de usabilidad. De hecho, el programa Mview tiene la opción de agrandar el modelo molecular (zoom), la cual utilizaron todos los estudiantes.

2. ¿Los estudiantes podrán interactuar de manera satisfactoria con la estructura molecular mostrada en el teléfono inteligente?

De acuerdo a los resultados del cuestionario SUS, 95\% de los estudiantes se sintieron en confianza y satisfechos de haber utilizado el visualizador Mview en el teléfono inteligente.

3. ¿El visualizador molecular corriendo en el teléfono inteligente será lo suficientemente eficaz y eficiente para mostrar la molécula a estudiar?

De acuerdo al método de Protocolo de Pensamiento Manifestado utilizado en el estudio de usabilidad, se vio que el estudiante, por medio del visualizador Mview, abrió y mostró en la pantalla los tres modelos moleculares utilizados en el estudio con eficacia y eficiencia. Además, el programa Mview y el teléfono inteligente fueron lo suficientemente eficientes para realizar los cálculos de los ángulos y medición de enlaces sin retraso alguno.

4. ¿Los estudiantes estarán interesados en seguir utilizando el visualizador molecular en el teléfono inteligente en futuras clases?

Sí, ya que de acuerdo a los resultados del cuestionario SUS, 95\% de los participantes desean seguir utilizando la visualización molecular en teléfonos inteligentes en futuras clases de química. Por otro lado, de acuerdo al cuestionario de actividades sobre los tres sistemas moleculares analizados, se obtuvieron los siguientes resultados:

- El $100 \%$ de los alumnos midieron correctamente las longitudes de enlace C-C y C-H de la molécula del etano. Pero en la medición de los ángulos, tanto de enlace como diedros, solamente el $70 \%$ de los alumnos lo determinaron correctamente. De este porcentaje, el $73 \%$ corresponde a los varones y el $27 \%$ a las mujeres.

- El 68\% de los alumnos asignaron correctamente la configuración (S) del centro quiral de la alanina según el método propuesto por Cahn, Ingold y Prelog. De este porcentaje el 77\% correspondió a las mujeres y el $56 \%$ a los varones.

- La última actividad que consistió en calcular el número de pentágonos que posee el $\mathrm{C}_{60}$ (buckyball) el $55 \%$ de los alumnos lo determinaron correctamente. De este porcentaje, el 67\% correspondió a los varones y el $46 \%$ a las mujeres.

Estos resultados muestran algunos aspectos interesantes, por ejemplo la determinación de la longitud de enlace C-C y C-H es trivial puesto que solo hay dos carbonos y tampoco hay confusión en el enlace C-H; pero parece que en la determinación de los ángulos de enlace y diedros los alumnos no tuvieron el suficiente cuidado en la selección consecutiva del ángulo de enlace $\mathrm{C}-\mathrm{C}-\mathrm{H}$, pudiendo haber seleccionado erróneamente un ángulo $\mathrm{C}-\mathrm{H}-\mathrm{C}$ por ejemplo. El caso del ángulo diedro es un poco más complicado por necesitar cuatro puntos y es fácil que el alumno haya incurrido en errores de selección atómica.

Tabla 2. Resultados de las 10 escalas de Likert del cuestionario SUS

\begin{tabular}{|c|c|c|c|c|}
\hline \# de escala: & Escala & Promedio & Moda & Desviación estándar \\
\hline 1 & Me gustaría utilizar con frecuencia el programa Mview & 4.86 & 5 & 0.47 \\
\hline 2 & Encontré el programa Mview innecesariamente complejo & 2.38 & 1 & 1.56 \\
\hline 3 & Fue fácil utilizar el Mview & 4.95 & 5 & 0.21 \\
\hline 4 & Creo que necesitaría del apoyo de un experto para utilizar el Mview & 2.05 & 1 & 1.43 \\
\hline 5 & Las diversas funciones del Mview están bien integrados & 4.57 & 5 & 0.66 \\
\hline 6 & Hubo demasiada inconsistencia en el Mview & 1.76 & 1 & 1.31 \\
\hline 7 & Imagino que la mayoría de las personas aprenderían muy rápidamente a utilizar el Mview & 4.90 & 5 & 0.29 \\
\hline 8 & Encontré el Mview muy difícil de usar. & 1.55 & 1 & 1.24 \\
\hline 9 & Me sentí muy confiado en la navegación del Mview. & 4.76 & 5 & 0.43 \\
\hline 10 & Necesito aprender muchas cosas antes de utilizar el Mview & 1.86 & 1 & 1.04 \\
\hline
\end{tabular}


A pesar de que los alumnos ya han cursado materias de química donde aprendieron a asignar las configuraciones espaciales $(R)-(S)$ de moléculas específicas, los resultados muestran no una falta de pericia en el uso del dispositivo móvil sino una falta de práctica constante y de experiencia en la visualización 3D de sistemas moleculares.

Es curioso observar en los resultados del número de pentágonos del buckyball, que fueron los varones quienes obtuvieron una ligera ventaja de asertividad (55\%) con respecto a las mujeres. Este resultado, podría decirnos que aparentemente los varones tienen una mejor habilidad espacial rotacional que las mujeres, lo que ya se ha sugerido en otros reportes. ${ }^{27,28}$

\section{CONCLUSIONES}

Después de analizar los resultados del estudio de usabilidad, encontramos las siguientes ventajas educativas con respecto a utilizar modelos moleculares mostrados en teléfonos inteligentes: complementar otras formas de aprendizaje utilizadas en el aula de clase; mejorar la comprensión de conceptos imposibles de ver a simple vista; usar representaciones para comunicar conceptos a compañeros y profesores; recordar más fácilmente temas que involucran datos, fórmulas o características específicas; determinar los tipos de enlaces (sencillos, dobles o triples) que tiene una molécula; activar o desactivar la rotación de moléculas en tres dimensiones para apreciar los ángulos de los enlaces; medir ángulos en una molécula para determinar su forma (lo que a su vez determina la función); establecer relaciones visuales entre modelos moleculares en dos y tres dimensiones; comparar simultáneamente diferentes representaciones moleculares (esferas y barras, barras, modelo compacto, etc); manipular sustancias en laboratorios virtuales antes de hacerlo físicamente (en algunos casos por seguridad) y sin incurrir en gastos; relacionar visualmente las propiedades de una molécula con la experiencia física del laboratorio; facilidad de acceder a la visualización molecular en cualquier lugar y en cualquier momento (anywhere-anytime) en línea y utilizando redes inalámbricas. Por ejemplo, un profesor de química puede explicar de manera rápida algún concepto de la estructura de una molécula a un alumno estando ambos fuera de clase en el pasillo o en la cafetería de la escuela; aceptabilidad de parte de muchos usuarios de computación y telefonía móvil para acceder a los modelos moleculares; los programas de visualización molecular generalmente son de bajo costo o gratuitos, ya que algunos son del tipo open source; la visualización molecular en dispositivos móviles puede ser útil para aprender conceptos generales de una estructura molecular; la visualización molecular en dispositivos móviles puede ayudar a complementar la lectura de un libro de bioquímica; la mayoría de los visualizadores pueden abrir archivos de moléculas en formato Protein Data Bank (PDB), los cuales se bajan de repositorios de libre acceso, por lo que los estudiantes pueden acceder y visualizar muchos tipos de moléculas.

Sin embargo, la visualización molecular en teléfonos inteligentes puede presentar estos retos: el tamaño de la pantalla de los dispositivos móviles puede ser una limitante para visualizar ciertas macromoléculas; los visualizadores moleculares actuales para los teléfonos inteligentes (tal como el Mview) tienen un número reducido de funciones; la mayoría de los visualizadores moleculares de dispositivos móviles (como los mostrados en la Tabla 1) están limitados a abrir y desplegar moléculas relativamente pequeñas, debido a las características del hardware. Por ejemplo, el programa Mview puede mostrar moléculas que contengan hasta 240 átomos.

Habiendo encontrado lo anterior, consideramos que el uso de teléfonos inteligentes para la comprensión de la información molecular de moléculas pequeñas es adecuado y pertinente a las aplicaciones y tendencias actuales de cómputo móvil en ambientes educativos, debido a su actual usabilidad (eficacia, eficiencia y satisfacción de uso).

De acuerdo a lo encontrado en las evaluaciones de usabilidad de los estudiantes de química en nuestro experimento, consideramos que la visualización molecular en teléfonos inteligentes ofrece las mismas aplicaciones educativas que se encuentran en visualizadores moleculares de computadoras de escritorio utilizados de manera regular en clases de química y aún más, ya que la visualización molecular en teléfonos incluye las ventajas de movilidad, donde los estudiantes pueden analizar una molécula en cualquier lugar y en cualquier momento, permitiendo analizar y aprender aspectos generales de la forma y función de una molécula de manera rápida y práctica. Este tipo de visualización, al igual que la visualización molecular en computadoras de escritorio, es una herramienta útil que fomenta las habilidades de aprendizaje necesarias para la comprensión de estructuras moleculares, tales como la inspección libre de una molécula incluyendo su rotación, traslación y ampliación, mediciones de enlaces, cálculo de ángulos entre átomos, visualización con diversos formatos, entre otros.

El teléfono móvil se encuentra entre los medios favoritos de los jóvenes. La franja de edad entre 10 y 18 años se caracteriza por ser la primera generación que ha crecido con éste medio y por lo tanto lo ve totalmente integrado a su propia vida, por lo tanto, un software de química instalado en los teléfonos móvil inteligentes podría ser un recurso educativo que capta la atención de un estudiante ya que se utiliza un medio en donde un joven más se ha desarrollado con lenguajes similares, además es un medio de aprendizaje relativamente de bajo costo.

Como trabajo a futuro, necesitaremos realizar más pruebas de usabilidad con otros modelos y marcas de teléfonos móviles inteligentes diferentes al utilizado en este estudio. Se necesitará también hacer pruebas con diferentes moléculas y hacer estudios longitudinales para así ver cómo se comporta el programa Mview y si esto afectará la interacción de los usuarios con el programa a largo plazo.

\section{AGRADECIMIENTOS}

Todas las marcas registradas y logotipos a los que se hicieron referencia en este artículo pertenecen a sus respectivas compañías. Parte de la investigación llevada a cabo para la elaboración de este artículo se realizó en el Laboratorio de Interacción Humano-Computadora de la Facultad de Telemática, perteneciente a la Universidad de Colima, México. Se agradece la participación de los estudiantes de la Facultad de Ciencias Químicas de la Universidad de Colima que participaron en el estudio de usabilidad.

\section{REFERENCIAS}

1. Nakhleh, M. B.; J. Chem. Educ. 1992, 69, 3.

2. Ben-Zvi, R.; Eylon, B. ; Silberstein, J.; J. Educ. Chem. 1987, 24, 4.

3. Ozmen, H.; J. Sci. Educ. Tech. 2004, 13.

4. Harle, M.; Towns, M.; J. Chem. Educ. 2011, 88, 3.

5. Durso, F. T.; Handbook of Applied Cognition, $2^{\text {nd }}$ ed., Wiley: New York, 2007.

6. Johnson, B. G.; Gill, P. M. W.; Pople, J. A.; Chem. Phys. Lett. 1993, 206.

7. Casher, O.; Leach, C.; Page, C. S.; Rzepa, H. S.; Chemistry in Britain 1998, 34, 26.

8. Berman, H. M.; Acta Crystallogr., Sect. A: Found. Crystallogr. 2008, A64 (1).

9. Garcia-Ruiz, M. A.; Bustos Mendoza, C.; Andrade Arechiga, M.; Acosta Diaz, R.; Revista de Educación Quimica 2006, 17, 1.

10. Garcia-Ruiz, M. A.; Valdez-Velazquez, L. L.; Gomez-Sandoval, Z.; Quim. Nova 2008, 31, 2184. 
11. Canning, D. R.; Cox, J. R.; Chemistry Education: Research and Practice in Europe 2001, 2, 2.

12. Williams, A. J.; Pence, H. E.; J. Chem. Educ. 2011, 88, 6.

13. Gilder, J. R.; Raymer, M.; Doom, T.; Abstracts, $2^{\text {nd }}$ IEEE International Symposium on Bioinformatics and Bioengineering, Philadelphia, EUA, 2001.

14. Paredes, J.; Pennington, R. L.; Pursell, D. P.; Sloop, J. C.; Tsoi, M. Y.; Georgia J. Sci. 2010, 68, 4.

15. Sauder, D.; Timpte, C.; Pennington, R.; Tsoi, M. Y.; Paredes, J. B.; Pursell, D. En Proceedings of World Conference on Educational Multimedia, Hypermedia and Telecommunications; G. Siemens, G.; Fulford, M. C., eds.; AACE: Chesapeake, 2009, cap. 3.

16. ISO 9241-11; Ergonomic requirements for office work with visual display terminals (VDTs) - Part 11: Guidance on usability, International Organization for Standardization, 1998.

17. MacFarlane, S.; Sim, G.; Horton, M.; Memorias de 2005 Conference on interaction Design and Children, Boulder, Colorado,2005.

18. Zaharias, P.; eLearn Magazine 2004, 2004, issue 6.
19. Zaharias, P.; Memorias de CHI 2006, Montreal, Canadá, 2006

20. Virvou, M.; Katsionis, G.; Computers \& Education 2008, 50.

21. Smith, S. P.; Hart, J.; Abstracts, IEEE Symposium on 3D User Interfaces, Alexandria, EUA, 2006

22. Brooke, J. En Usability Evaluation in Industry; Jordan, P. W.; Thomas, B.; Weerdmeester, B. A.; Mclelland, A.L., eds.; Taylor and Francis: Londres, 1996, cap. 2.

23. http://155.210.207.183/aipo/documentos/codigo_etico_AIPO.pdf, accedida Junio 2011.

24. Curl, R. F.; Smalley, R. E.; Sci. Am. 1991, 265.

25. Kroto, H. W.; Heath, J. R.; O’Brien, S. C.; Curl, R. F.; Smalley, R. E.; Nature 1985, 318, 6042 .

26. Boo, W. J. J.; Chem. Educ. 1992, 69, 605

27. Harris, L. J. En Asymmetrical Function of The Brain; Kinsbourne, M., ed.; Cambridge University Press: Cambridge, 1978, cap. 4.

28. Yong Yoo, H; Hyun Cheon, S.; Memorias de APVis 'O6 Proceedings of the 2006 Asia-Pacific Symposium on Information Visualisation, Australian Computer Society, Inc. Darlinghurts, Australia, 2006, vol. 60.

Anexo A. Cuestionario de usabilidad System Usability Scale (SUS) ${ }^{22}$

Favor de marca con una "X" en la columna correspondiente según sea tu criterio.

En completo de-

Completamente sacuerdo

de acuerdo

- Me gustaría utilizar con frecuencia el programa Mview

$(\quad)$

- Encontré el programa Mview innecesariamente complejo

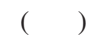

- Fue fácil utilizar el Mview

- Creo que necesitaría del apoyo de un experto para utilizar el( ) Mview

- Las diversas funciones del Mview están bien integrados

- Hubo demasiada inconsistencia (diferentes colores y fuentes $\sin (\quad$ ) armonía, etc.) en el Mview

- Imagino que la mayoría de las personas aprenderían muy rápi-( ） damente a utilizar el Mview

- Encontré el Mview muy difícil de usar.

- Me sentí muy confiado en la navegación del Mview.

- Necesito aprender muchas cosas antes de utilizar el Mview

$(\quad)$
$(\quad)$
$(\quad)$

